\title{
Procedimiento de selección de factores de influencia en el análisis de regresión ${ }^{(\bullet)}$
}

\author{
Method for selecting the influencing factors in regression analysis
}

\author{
J. Tošenovský ${ }^{(*)}$
}

En las aplicaciones del análisis de regresión ocurre, con frecuencia, que durante la elaboración del modelo, cuando se buscan los factores principales que influyen en el índice $y$, se incluyen en este prácticamente todos los factores $x_{1}, x_{2}, \ldots, x_{k}$ que pueden influir, aunque sea en pequeña medida.

$\mathrm{Al}$ mantener todas esas variables puede suceder que el modelo resultante no sólo sea complicado, sino que, sobre todo, sea incorrecto.

Por esta razón, es deseable hacer una selección preliminar para obtener un modelo funcional que, al mismo tiempo, sea sencillo y correcto. En este artículo se trata de mostrar una posible selección.

\section{ALGORITMO PARA LA SELECCIÓN DE LOS FACTORES SIGNIFICANTES}

Con frecuencia, como modelo inicial se considera la función

$$
y=\beta_{1} \cdot x_{1}+\beta_{2} \cdot x_{2}+\ldots+\beta_{k} \cdot x_{k}
$$

$\mathrm{Al}$ decidir qué factores (variables) del conjunto $\left\{x_{1}, x_{2}, \ldots, x_{k}\right\}$ tienen que incluirse en el modelo [1], se puede proceder según los siguientes pasos:

a) Se forma el vector de las correlaciones entre $y$ y cada una de las variables $X_{1}, x_{2}, \ldots, x_{k}$ :

$$
R_{0}^{T}=\left(r_{y, x_{1}}, r_{y, x_{2}}, \ldots, r_{y, x_{k}}\right)=\left(r_{1}, r_{2}, \ldots, r_{k}\right)
$$

b) Se forma la matriz de las correlaciones entre los pares de las variables independientes $x_{1}, \ldots, x_{k}$ :

$(\bullet$ Trabajo recibido el día 5 de junio de 1996.

(*) Universidad Técnica en Ostrava. República Checa.

$$
R=\begin{array}{cccc}
r_{11} & r_{12} & \ldots & r_{1 k} \\
r_{21} & r_{22} & \ldots & r_{2 k} \\
\ldots & \ldots & \ldots & \ldots \\
r_{k 1} & r_{k 2} & \ldots & r_{k k}
\end{array}
$$

c) Se calcula el valor crítico del coeficiente de correlación $r^{*}$ :

$$
r^{*}=\frac{t_{n-2}(\alpha)}{\sqrt{t_{n-2}(\alpha)+n-2}}
$$

donde $t_{n-2}(\alpha)$ es el valor crítico de la distribución de Student y se busca en las tablas correspondientes; $\alpha$ es el nivel de significación.

Después de esto se procede a realizar la selección, que consta de dos etapas según la siguiente "filosofía": el coeficiente de correlación, $r_{i}$, que no es suficientemente grande, indica que $y$ no depende notablemente de $x_{i}$ y por eso se retira. El término "notablemente" significa mayor que $r^{*}$. De esta forma, se consigue otro punto del algoritmo:

d) Excluir esas $x_{i}$ de [1] para las cuáles

$$
\left|r_{y, x_{i}}\right|=\left|r_{i}\right| \leq r^{*}
$$

Entre el resto de $r_{i}$ se busca el mayor coeficiente de correlación, por ejemplo, $r_{k}$, para el que vale

$$
\left|r_{k}\right|=\max _{i}\left\{\left|\mathrm{r}_{\mathrm{i}}\right|\right\}
$$

y se busca la correlación entre $x_{k}$ y las demás variables.

Las variables que tienen con $x_{k}$ una correlación mayor que $r^{*}$ se retiran de [1], porque su influencia sobre $y$ está originada por una gran correlación con el factor de influencia más significativa, $x_{k}$.

Por eso, el último paso del algoritmo es: 
e) Retirar esas variables $x_{i}$ de [1] para las cuales

$$
r^{*}<\left|r_{k, i}\right|
$$

\section{Ejemplo}

El uso del algoritmo citado se muestra en el siguiente problema, que trata sobre la relación entre la resistencia del coque y los factores elegidos.

Se supone, en primer lugar, que la resistencia del coque, $y$, depende de los siguientes factores:

$$
y=f\left(x_{1}, x_{2}, \ldots, x_{10}\right)
$$

$x_{1}=$ ceniza en el coque

$x_{2}=$ resistencia del coque al desgaste por fricción en frío

$x_{3}=$ resistencia del coque en frío

$x_{4}=$ dilatación total

$x_{5}=$ dilatación del carbono

$x_{6}=$ contenido de la materia inflamable superficial en el carbón

$x_{7}=$ contenido de ceniza en el carbón

$x_{8}=$ temperatura última de coquefacción

$x_{9}=$ humedad del coque

$x_{10}=$ reactividad del coque

a) Se construyen las matrices necesarias:

$$
\begin{gathered}
R_{0}^{T}=(0,38,-0,45,0,46,0,62,0,61,-0,48, \\
0,50,-0,27,-0,28,-0,91)
\end{gathered}
$$

b)

$$
\begin{array}{ccccccccccc}
x_{1} & x_{2} & x_{3} & x_{4} & x_{5} & x_{6} & \multicolumn{1}{c}{x_{7}} & x_{8} & \multicolumn{1}{c}{x_{9}} & x_{10} & \\
1 & 0,28 & 0,32 & -0,49 & 0,43 & 0,05 & -0,70 & -0,58 & 0,24 & -0,39 & x_{1} \\
& 1 & 0,37 & -0,08 & 0,07 & -0,20 & -0,24 & -0,14 & 0,20 & -0,31 & x_{2} \\
& & 1 & 0 & -0,03 & 0,47 & 0 & 0,08 & 0,09 & 0,03 & x_{3} \\
& \mathrm{R}= & & 1 & -0,49 & 0,06 & 0,48 & 0,81 & -0,53 & 0,48 & x_{4} \\
& & & 1 & 0,06 & -0,44 & -0,77 & 0,53 & -0,46 & x_{5} \\
& & & & 1 & 0,30 & 0,20 & -0,19 & 0,08 & x_{6} \\
& & & & & 1 & 0,65 & -0,26 & 0,52 & x_{7} \\
& & & & & & 1 & -0,61 & 0,56 & x_{8} \\
& & & & & & & 1 & -0,37 & x_{9}
\end{array}
$$

c)

$$
\begin{gathered}
r^{*}=\frac{t_{n-2}(\alpha)}{\sqrt{t_{n-2}(\alpha)+n-2}}= \\
=\frac{t_{30-2}(0,05)}{\sqrt{t_{30-2}(0,05)+30-2}}=0,374
\end{gathered}
$$

d) Menores que $r^{*}$ son en $R_{0}$ los coeficientes de correlación $r_{8}$ y $r_{9}$; por eso se retiran las variables $x_{8}$ y $x_{9}$.
La correlación más fuerte con $y$ la tiene, en $R_{0}$, el factor $x_{10}$.

e) Las variables que tienen una correlación con $y$ mayor que $r^{*}$ son: $x_{1}, x_{4}, x_{5}$ y $x_{7}$.

$\mathrm{Al}$ retirar estas variables del modelo [1] quedan

$$
y=f\left(x_{2}, x_{3}, x_{6}, x_{10}\right)
$$

la que se considera, según [1] en la forma

$$
y=\beta_{2} \cdot x_{2}+\beta_{3} \cdot x_{3}+\beta_{6} \cdot x_{6}+\beta_{10} \cdot x_{10}
$$

Ahora, la estimación $\beta_{2}, \beta_{3}, \beta_{6}$ y $\beta_{10}$ se realizará sin problemas utilizando la fórmula bien conocida

$$
\hat{\beta}=\left(X^{T} X\right)^{-1} \cdot X^{T} Y
$$

al contrario de la situación, en la que se consideran todos los factores.

El problema antes mencionado se basa en la búsqueda de la matriz inversa en [2]: si en la matriz $X$ hay dos columnas correlacionadas, o no es posible encontrar la matriz inversa o existe, pero la estimación de los coeficientes tiene una dispersión enorme. Esto puede suceder en los casos en que en el modelo se incluyen todos los factores, sin que se realice la selección antes mencionada.

Es verdad que hay situaciones en las que en la matriz $X$ hay columnas correlacionadas, que según las condiciones de los mínimos cuadrados ordinarios no son admisibles, pero no es posible prescindir de ellas, ya que cada una de ellas es indispensable para el modelo. En esos casos, en [2] se utiliza, en lugar de la matriz inversa $\left(X^{T} \cdot X\right)^{-1}$, otro procedimiento.

$$
\left(X^{T} \cdot X\right)^{-1}=\sum_{i=1}^{k} \lambda_{i}^{-1} P_{i} P_{i}^{T}
$$

donde $\quad \lambda_{i}=$ núm. característico de la matriz $X^{T} X$ $P_{i}=$ vector característico de la matriz $X^{T} X$

si

$$
\left|\frac{\lambda_{i}}{\lambda_{i}} \lambda_{i=1}\right| \leq p
$$

entonces se retira $\lambda_{i}$ $p=$ núm. bastante pequeño, por ejemplo $p=10^{-15}$.

\section{BIBLIOGRAFÍA}

NowAK, E. Zarys metod ekonometrii. Wydawnictvo naukove. Varsovia (Polonia), 1990: 19-22. 
Draper, N. y Smith, H. Applied Regression Analysis. John Wiley and Sons. Nueva York (EE.UU.), 1981.

Meloun, M. y Militky, J. Statistické zpracování experimentálních dat. Plus. Praga (R. Checa), 1994.

GutTMAn, I. Linear models-An Introcution. John Wiley and Sons. Nueva York (EE.UU.), 1982.

MARQUART, D.M. Technometrics, 12, 1970: 591.

AtKinson, A.C. Plot, Transformation, Regression. Claredon Press. Oxford (R.U.), 1986.

Rousseuw, P.J. y LEROY A.M. Robust Regression and Outliers Detection. John Wiley and Sons. Nueva York (EE.UU.), 1987.
NASZODI, L.J. Technometrics, 20, 1978: 201.

CooK, R.D. y Weisberg, S. Biometrika, 71, 1983: 1.

MaLows, C.L. Technometrics, 28, 1986: 313.

JOINER, B. Amer. Statist., 35, 1981: 227.

RICE, J.A. Mathematical Statistics and Data Analysis. Wadsworth and Brooks. California (EE.UU.), 1988.

KLEInBAum, D.G. Applied Regression Analysis and Other Multivariate Methods. PSW-KENT Publishing Comp. Boston (EE.UU.), 1988.

Himmelblau, D. Process Analysis by Statistical Methods. John Wiley \& Sons. Nueva York (EE.UU.), 1969. 